\title{
COMMENTARY
}

\section{Oxidative stress in the brain and arterial hypertension}

\author{
Ruy R Campos \\ Hypertension Research (2009) 32, 1047-1048; doi:10.1038/hr.2009.180; published online 6 November 2009
}

A ngiotensin II (Ang II) acting in the brain has been implicated in the pathogenesis of many cardiovascular diseases, such as arterial hypertension. One of the most important consequences of activation of the Ang II type 1 (AT1) receptor is enhanced nicotinamide adenine dinucleotide phosphate (NADPH) oxidase activity, eliciting the production of reactive oxygen species (ROS), especially the superoxide anion $\left(\mathrm{O}_{2}{ }^{-}\right) \cdot{ }^{1-3}$ The close functional association between NADPH oxidase and the renin-angiotensin system may be of particular relevance in linking oxidative stress to hypertension. The brain is one of the Ang II targets most affected by oxidative stress. In fact, the central nervous system is involved in the development and maintenance of Ang II-dependent arterial hypertension. ${ }^{4}$ The rostral ventrolateral medulla (RVLM) and the hypothalamic paraventricular nucleus (PVN) are the major regions related to sympathetic activation during the development of arterial hypertension. ${ }^{4}$ Recently, we showed that AT1 gene expression receptors are upregulated in the RVLM and PVN areas of renovascular hypertensive rats. ${ }^{5}$ Furthermore, expression of NADPH oxidase subunits ( $p 47$ phox and $g p$ 91 phox) was increased in the PVN and RVLM areas of renovascular hypertensive rats. Tempol administration into the PVN or RVLM decreased blood pressure and renal sympathetic nerve activity only in hypertensive rats, suggesting that renal sympathetic hyperactivity and hypertension are associated with an increase in central oxidative stress. One possible mechanism by which circulating Ang II exerts its effects centrally is by binding to its receptors on neurons in specialized brain regions that lack a blood-

Dr RR Campos is at the Cardiovascular Division, Department of Physiology, Federal University of São Paulo, Rua Botucatu, 862, Sao Paulo 04023-060, Brazil.

E-mail: campos@fcr.epm.br brain barrier, that is, the circumventricular organs (CVOs). The CVOs are populated with fenestrated capillaries for high permeability and are known to be pivotal in mediating the increase in blood pressure in response to circulating Ang II. Neurons in these regions, such as the subfornical organ and the organum vasculosum of the lamina terminalis, can be activated by circulating Ang II and they then signal to other downstream cardiovascular control regions of the brain, such as the RVLM and the PVN, which further activate several neurohumoral pathways (Figure 1). However, the exact mechanism by which circulating Ang II leads to an increase in oxidative stress in the sympathetic premotor neurons is unclear. Paterson et al. ${ }^{6}$ showed that gp91phox is abundantly expressed in CVOs and identified gp91phox as having a key role in central Ang II-induced cardiovascular responses by increasing ROS production, suggesting a strong relationship between upregulation of mRNA NADPH subunits, particularly gp91phox and p47phox, and increased oxidative stress in the central nervous system in response to circulating Ang II. In an interesting paper, Chan et al. ${ }^{7}$ demonstrated that phosphorylation of p47phox serine residues induced by Ang II in the RVLM, which was blunted by the AT1 receptor antagonist losartan, manifested a temporal profile that correlated positively with the $\mathrm{O}_{2}$ production induced by Ang II. Therefore, these results could imply that enhanced central Ang II action stimulates NADPH oxidase in the central nervous system, leading to increased $\mathrm{O}_{2}$ formation and thereby an increase in sympathetic activity and arterial hypertension. Furthermore, Kishi et al. ${ }^{8}$ showed that thiobarbituric acid-reactive substance levels were increased in the RVLM of spontaneously hypertensive rats (SHRs) compared with Wistar-Kyoto rats. Tempol injected into this region decreased blood pressure in a dose-dependent manner in the hypertensive group but not in the control group, suggesting that in this model central oxidative stress is a major mechanism for maintained hypertension. Therefore, it is plausible that chronic Ang II AT1 receptor antagonist treatment in hypertension decreases central oxidative stress and blood pressure. Interestingly, the paper from Araki et al. ${ }^{9}$ showed that chronic olmesartan treatment in stroke-prone SHR (SHRSP) rats decreased oxidative stress in the brain and norepinephrine excretion. More importantly, they showed that the same reduction in blood pressure afforded by hydralazine/hydrochlorothiazide (Hyd/HCT) did not reduce brain oxidative stress, suggesting that a specific antioxidant effect in the brain was mediated by the AT1 receptor antagonist; this effect seemed to be independent of the reduction in blood pressure. Finally, they showed that the decrease in blood pressure in response to olmesartan, unlike Hyd/HCT, did not induce a baroreflexmediated increase in HR and sympathetic activity despite similar reductions in blood pressure, indicating that olmesartan has an anti-oxidative role in the brain without stimulating reflex-mediated sympathetic activity in the SHRSP. These experiments shed new light on the importance of the central effects of Ang II and oxidative stress in the brain in arterial hypertension and sympathoexcitation. However, the source of superoxide formation in regions of the brain involved in cardiovascular control has not been determined; whether AT1 receptor activation can specifically increase ROS formation in cardiovascular or non-cardiovascular neurons or in glial cells or in endothelial cells remains to be established. Thus, longer-term activation of the AT1 receptor in the brain may have the potential for deleterious positive-feedback effects of Ang II's actions on the central nervous system, and this may play a major role in hypertension. Elucidating the mechanism involved in the central sympathoexcitation 


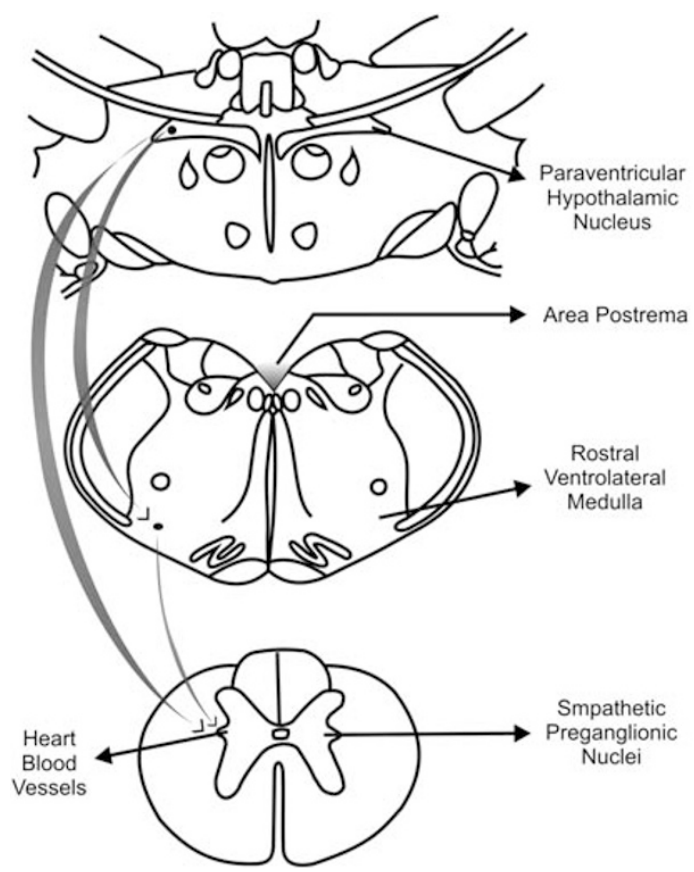

\section{Increased circulating \\ Angiotensin II - acting via \\ $\mathrm{AT}_{1}$ receptors in the}

circunventricular organs.

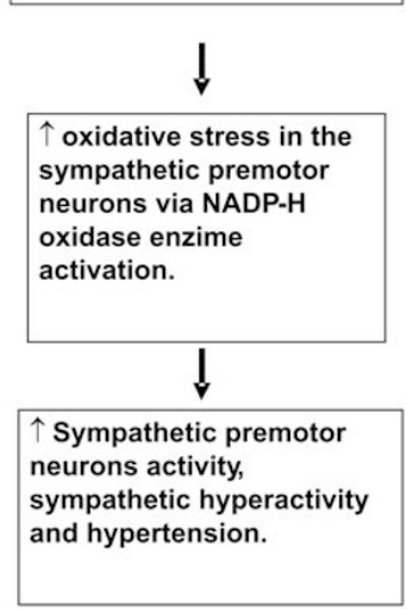

Figure 1 Schematic representation of the possible central pathways involved in the sympathetic activation induced by increases in central oxidative stress in response to an increase in circulating angiotensin II.

mediated by Ang II and oxidative stress may result in a new paradigm for treating arterial hypertension. However, further integrative and molecular studies are necessary for a better understanding of the balance between peripheral and central oxidative stress and its contribution to arterial hypertension.

1 Berry C, Hamilton CA, Brosnan MJ, Magill FG, Berg GA, McMurray JJ, Dominiczak AF. Investigation into the sources of superoxide in human blood vessels: angiotensin II increases superoxide production in human internal mammary arteries. Circulation 2000; 101: 2206-2212. 2 Griendling KK, Minieri CA, Ollerenshaw JD, Alexander RW. Angiotensin II stimulates NADH and NADPH oxidase activity in cultured vascular smooth muscle cells. Circ Res 1994; 74: 1141-1148.

3 Zimmerman MC, Dunlay RP, Lazartigues E, Zhang Y, Sharma RV, Engelhardt JF, Davisson RL. Requirement for Rac1-dependent NADPH oxidase in the cardiovascular and dipsogenic actions of angiotensin II in the brain. Circ Res 2004; 95: 532-539.

4 Campos RR, Bergamaschi CT. Neurotransmission alterations in central cardiovascular control in experimental hypertension. Curr Hypertens Rev 2006; 2: 193-198.

5 Oliveira-Sales EB, Nishi E, Carillo BA, Boim MA, Dolnikopf MS, Bergamaschi CT, Campos RR. Oxidative stress in the sympathetic premotor neurons contributes to sympathetic activation in renovascular hypertension. Am J Hypertens 2009; 22: 484-492.

6 Peterson J, Burmeister MA, Tian X, Stupinski JA, Sharma VA, Davisson RL. Both Nox2- and Nox4-containing $\mathrm{NAD}(\mathrm{P}) \mathrm{H}$ oxidases are required for the full vasopressor effects of angiotensin-II (Ang-II) in the central nervous system. FASEB J 2007; 21: 752.1.

7 Chan SH, Hsu KS, Huang CC, Wang LL, Ou CC, Chan JY. NADPH oxidase-derived superoxide anion mediates angiotensin II-induced pressor effect via activation of p38 mitogen-activated protein kinase in the rostral ventrolateral medulla. Circ Res 2005; 97 : 737-739.

8 Kishi T, Hirooka Y, Kimura Y, Ito K, Shimokawa H, Takeshita A. Increased reactive oxygen species in rostral ventrolateral medulla contribute to neural mechanisms of hypertension in stroke-prone spontaneously hypertensive rats. Circulation 2004; 09 : 2357-2362.

9 Araki S, Hirooka Y, Kishi T, Yasukawa K, Utsumi H, Sunagawa K. Olmesartan reduces oxidative stress in the brain of stroke-prone spontaneously hypertensive rats assessed by an in vivo ESR method. Hypertension Res 2009; 32: 1091-1096. 